\title{
Parastomal hernia repair
}

\author{
Jeffrey Michael Smith ${ }^{1,2}$, Sahil K. Kapur ${ }^{1}$, Alexander F. Mericli ${ }^{1}$, Donald P. Baumann ${ }^{1}$, Charles E. Butler ${ }^{1}$ \\ 'Department of Plastic Surgery, The University of Texas MD Anderson Cancer Center, Houston, TX 77030, USA. \\ 2Department of Surgery, Division of Plastic Surgery, The University of Texas Medical Branch, Galveston, TX 77555, USA.
}

Correspondence to: Dr. Sahil K. Kapur, Department of Plastic Surgery, Unit 1488, The University of Texas MD Anderson Cancer Center, 1400 Pressler St., Houston, TX 77030, USA. E-mail: skkapur@mdanderson.org

How to cite this article: Smith JM, Kapur SK, Mericli AF, Baumann DP, Butler CE. Parastomal hernia repair. Plast Aesthet Res 2022;9:10. https://dx.doi.org/10.20517/2347-9264.2021.48

Received: 19 May 2021 First Decision: 23 Aug 2021 Revised: 27 Nov 2021 Accepted: 20 Dec 2021 Published: 8 Feb 2022

Academic Editors: Karol A. Gutowski, Marten Basta Copy Editor: Xi-Jun Chen Production Editor: Xi-Jun Chen

\begin{abstract}
Despite significant advances in abdominal wall reconstruction, parastomal hernias remain a complex problem, with a high risk of recurrence following repair. While a number of surgical hernia repair techniques have been proposed, there is no consensus on optimal management. Several clinical variables must be considered when developing a comprehensive repair plan that minimizes the likelihood of hernia recurrence and surgical site occurrences. In this review, we describe the incidence of parastomal hernias and discuss pertinent risk factors, medical history findings, physical examination findings, supplementary diagnostic modalities, parastomal hernia classification systems, surgical indications, and repair techniques. Special consideration is given to the discussion of mesh reinforcement, including available biomaterials, anatomic plane selection, and the extent of mesh reinforcement. Although open repairs are the primary focus of this article, minimally invasive laparoscopic and robotic approaches are also briefly described. It is our hope that the provided surgical outcome data will help guide surgical management and optimize outcomes for affected patients.
\end{abstract}

Keywords: Abdominal wall reconstruction, parastomal hernia, stoma, biologic mesh, synthetic mesh, polypropylene, acellular dermal matrix

\section{INTRODUCTION}

Parastomal hernias are defined as abnormal protrusions of the intra-abdominal contents through an abdominal wall defect, adjacent to or within the site of stoma creation. Parastomal hernias are a common 
complication following stoma creation, with incidences of $48.1 \%$ and $28.3 \%$ following colostomy and ileostomy, respectively ${ }^{[1]}$. However, on the basis of Pascal's principle of hydrostatic pressure and the law of Laplace, all stomata may eventually develop parastomal hernias ${ }^{[2]}$. Defining the true incidence of parastomal hernias is difficult because of varied follow-up times, non-standardized clinical definitions, and the asymptomatic presentation of these defects in many patients ${ }^{[3]}$.

Despite advances in the field of abdominal wall reconstruction, no consensus exists regarding the optimal management of stomas. A thorough understanding of the strengths and weaknesses of available surgical options is important to increase the probability of reconstructive success.

In this review, we describe the incidence of parastomal hernias and discuss pertinent risk factors, medical history findings, physical examination findings, supplementary diagnostic modalities, parastomal hernia classification systems, surgical indications, and repair techniques. Special consideration is given to the discussion of mesh reinforcement, including available biomaterials, anatomic plane selection, and the extent of mesh reinforcement. Although open repairs are the primary focus of this article, minimally invasive laparoscopic and robotic approaches are also briefly described. It is our hope that the provided surgical outcome data will help guide surgical management and optimize outcomes for affected patients.

\section{RISK FACTORS}

Patient-associated risk factors for developing parastomal hernias mirror those of other abdominal wall pathologies and include age > 60 years, female gender, obesity, hypertension, diabetes, smoking, corticosteroid use, elevated intra-abdominal pressure (e.g., constipation, benign prostatic hypertrophy, and ascites), chronic obstructive airway disease, collagen synthesis disorders, and malignancies ${ }^{[4-6]}$. Surgical risk factors at the time of stoma creation that confer an increased risk of eventual parastomal hernia formation include emergent indication, inappropriate stoma location, extensive fascial dissection, oversized fascial trephine, and intraperitoneal tunneling ${ }^{[7,8]}$.

The optimal site of stoma location is controversial. In a retrospective review of 65 patients, Sjödahl et al. ${ }^{[9]}$ demonstrated that stoma placement through the rectus abdominus muscle is associated with a lower rate of parastomal hernia development than its placement lateral to the rectus ( $26.1 \%$ vs. $2.8 \%$, no odds ratio or $P$ value available), yet this difference in risk remains disputed, even though trans-rectus stoma creation is traditionally recommended ${ }^{[10]}$. A recent abstract published by Nguyen et al. ${ }^{[11]}$ of 111 patients undergoing anterior cystectomy and vertical rectus abdominus musculocutaneous flap reconstruction found that parastomal hernia rates were equivalent for ileal conduits that were placed through the lateral abdominal wall or through intact rectus; however, these rates were much higher if the conduits were placed through an empty rectus sheath. Stoma placement within the linea semilunaris should be avoided because of potential destabilization of the abdominal wall.

Extraperitoneal tunneling of the colostomy to a hole in the abdominal wall has been associated with a lower rate of parastomal hernia development than has intraperitoneal tunneling [odds ratio $(\mathrm{OR})=0.41 ; 95 \%$ confidence interval $(\mathrm{CI}): 0.23-0.73 ; P=0.002]^{[12]}$. Extraperitoneal tunneling has been attributed to a more even distribution of pressure and tension on the abdominal wall than the single focal defect through all layers of the abdominal wall that is created by an intraperitoneal ostomy ${ }^{[13]}$. Prophylactic mesh reinforcement at the time of initial stoma creation has been demonstrated to result in a 4-fold reduction in the risk of developing a parastomal hernia $(\mathrm{OR}=0.24 ; 95 \% \mathrm{CI} \text { : } 0.12-0.50 ; P<0.001)^{[14]}$. A double-blind, randomized controlled trial demonstrated that the use of synthetic mesh to prophylactically reinforce a newly created colostomy does not significantly decrease the risk of hernia recurrence or have any significant 
impact on wound healing complications, with a median follow-up of one year ${ }^{[15]}$. A more recent randomized controlled trial with a 3-year median follow-up duration, however, demonstrated a lower parastomal hernia incidence in patients undergoing prophylactic mesh reinforcement of their ileal-urinary conduits ( $11 \% v s$. $23 \%, \mathrm{OR}=0.45 ; P=0.02)^{[16]}$.

\section{DIAGNOSIS}

The diagnosis of parastomal hernia is complicated because most patients are asymptomatic. Symptoms generally include pain, appliance leakage, maceration around the ostomy site, protrusion, cosmetic concerns, bowel obstruction, or symptoms of incarceration ${ }^{[7,17,18]}$. Physical examination involves careful palpation for a peri-stomal bulge or fascial defect while the patient performs the Valsalva maneuver. However, the diagnosis of parastomal hernia solely on the basis of clinical history and physical examination can be challenging, even for experienced clinicians. Obesity, severe pain during palpation, contracted scars on the abdominal skin, coexisting hernias along the laparotomy incision line, and neurogenic muscle relaxation can further complicate the detection of parastomal hernias ${ }^{[1]}$. Gurmu et al. ${ }^{[19]}$ found low interobserver diagnostic reliability on the basis of clinical examination alone, even among experienced colorectal surgeons with a special interest in parastomal hernia.

Consequently, imaging studies are often necessary to supplement clinical examination findings. In a 2020 systematic review of 29 studies comparing different modalities for the diagnosis of parastomal hernia, de Smet et al. ${ }^{[18]}$ reported that both computed tomography and ultrasonography are accurate imaging modalities, with an overall sensitivity of $83 \%$. Although computed tomography has greater diagnostic efficacy and inter-observer reliability, ultrasound may offer added benefits in terms of its dynamic nature, cost-effectiveness, and irradiation avoidance ${ }^{[18]}$. Furthermore, newer modalities, such as intrastomal 3D ultrasound, could alter the recommended diagnostic work-up of affected patients in the future. Further research is needed to validate these claims.

\section{CLASSIFICATION}

Early classification systems have been criticized for their lack of standardization and incorporation of lessrelevant variables ${ }^{[20]}$. Noting this, the European Hernia Society proposed a classification system to facilitate a comparative analysis of different studies ${ }^{[20]}$. To simplify classification, they determined that defect size (defined as the largest diameter of the hernia orifice in any direction), coexisting midline incisional hernia, and primary $v s$. recurrent status were the most relevant variables for classification. On the basis of these variables, four subclasses were defined: type I, small (diameter $<5 \mathrm{~cm}$ ) parastomal hernias with no central incisional hernia; type II, small (diameter $<5 \mathrm{~cm}$ ) parastomal hernias with central incisional hernia; type III, large (diameter $>5 \mathrm{~cm}$ ) parastomal hernia with no central incisional hernia; and type IV, large (diameter $>$ $5 \mathrm{~cm}$ ) parastomal hernia with central incisional hernia. These classifications can be further differentiated by primary vs. recurrent hernias. Since its inception in 2014, the European Hernia Society's classification system has been the most widely utilized system for parastomal hernias. It is also important to note that all parastomal hernias are classified as Ventral Hernia Working Group grade 3 hernias, which are predisposed to have a higher rate of recurrence, surgical site infection, and surgical site occurrence.

\section{SURGICAL MANAGEMENT}

The decision to pursue conservative management with attentive follow-up must be weighed against the potential risk of incarceration, strangulation, enlargement, poor appliance fit, and the development or worsening of medical comorbidities that may complicate future intervention ${ }^{[3]}$. Patients should be appropriately counseled and directed towards appropriate resources to optimize their medical and nutritional status. Patients may benefit from referral to an ostomy nurse for assistance with appliance 
leakage, fitting of supportive garments, and management of peristomal dermatitis ${ }^{[21]}$.

While there is a general agreement that strangulation, incarceration, and non-resolving obstruction necessitate urgent surgical intervention, there is less consensus regarding the less compelling indications for elective intervention, partly because of the relatively high risk of hernia recurrence [Table 1$]^{[1,7]}$. Factors that may warrant less urgent surgical correction include peristomal bulging, poor appliance fit, skin breakdown, pain, and aesthetic concerns ${ }^{[1,21]}$. Ultimately, patient preferences, surgeon experience, and relative risk must be considered when formulating an optimal treatment plan.

Additionally, the decision to proceed with surgical intervention must be weighed against the high rate of repair failure and recurrence. Historically high recurrence rates and unsatisfactory patient outcomes have driven innovation in the field of parastomal hernia repair. Common reconstructive options include primary suture repair, stoma relocation, and mesh-based repairs.

\section{Primary suture repair}

The oldest and least invasive method of parastomal hernia repair is directly closing the fascial defect with sutures. This technique involves making a peristomal cutaneous incision, reducing the hernia sac, and reapproximating the fascia using absorbable $v s$. non-absorbable heavy sutures. Although technical simplicity and laparotomy avoidance have been cited as benefits of direct repair, it is not recommended because of unacceptably high parastomal hernia recurrence rates, which range from $46 \%$ to $100 \%^{[3,8]}$. These high rates are likely explained by the inability to achieve tension-free closure and the baseline attenuation of the adjacent fascial tissues being used for the repair ${ }^{[22]}$. Although it is possible to reinforce a direct repair with mesh secured in the subcutaneous or pre-fascial spaces, the close proximity of the repair and the stoma presents a substantial risk of infection ${ }^{[23]}$. Given the aforementioned factors, direct suture repair is not routinely recommended and is often reserved for emergent cases or medically complex patients with short life expectancies who are unlikely to survive a more extensive operation ${ }^{[23]}$.

\section{Stoma relocation}

Relocation of the stoma to an alternative site on the abdominal wall may be considered in instances where the primary ostomy location is unsatisfactory; however, this technique has been largely abandoned in favor of alternative treatment modalities. The procedure can be performed both with and without a formal laparotomy ${ }^{[24]}$. The disadvantages of this technique include further weakening of the abdominal wall, high parastomal hernia recurrence rates, and the possible development of an incisional hernia at the previous ostomy site ${ }^{[1,25]}$. Furthermore, this approach may not be feasible in patients who have undergone multiple prior abdominal surgeries, especially if there is limited bowel length because of prior resection or adhesions. Repeated relocation also leads to a loss of available stoma sites. If a stoma is relocated, then the new stoma site should be reinforced with mesh or be located in a robust area of the abdominal wall. The prior stoma site should also be reinforced with mesh to reduce the risk of subsequent incisional hernia ${ }^{[26]}$.

\section{Mesh-based repairs}

Inspired by the success of prosthetic matrices in other types of abdominal wall reconstruction, Rosin and Bonardi ${ }^{[27]}$ introduced the use of reinforcing mesh for parastomal hernia repair in 1977. Since then, numerous mesh-based repairs have been developed that vary according to the type of mesh used, the anatomic placement, and the surgical technique (e.g., open mesh-based and minimally invasive meshbased) ${ }^{[28]}$. 
Table 1. Indications for parastomal hernia repair

\begin{tabular}{ll}
\hline Indications for required repair & Indications for elective repair \\
\hline Strangulation & Peristomal bulging \\
Incarceration & Poor appliance fit \\
Non-resolving obstruction & Skin breakdown \\
& Pain \\
& Aesthetic concerns \\
\hline
\end{tabular}

\section{Type of mesh}

Numerous different synthetic and biologic meshes have been utilized in the reconstruction of parastomal hernias. Macroporous synthetic meshes such as polypropylene and polyester possess high tensile strength and promote tissue ingrowth; however, although ingrowth yields additional mechanical strength, it can also promote significant complications, such as adhesions, bowel obstruction, and fistula ${ }^{[29]}$. Microporous meshes such as expanded polytetrafluoroethylene prevent tissue ingrowth but heal via encapsulation. Although these properties translate into a decreased risk of adhesion and erosion, they promote recurrence as a result of less structural stability and tissue incorporation. To maximize strength and minimize potential complications, composite meshes have been developed that combine macroporous and microporous elements. Although in the past, these were commonly constructed from a composite of polypropylene and expanded polytetrafluoroethylene, a number of other polymers have been utilized. Problems associated with synthetic meshes include infection, a need for explantation, and mesh contraction over time ${ }^{[29]}$.

In contrast to synthetic polymers, biologic meshes are vascularized and remodeled by the host tissues. These are often acellular matrices produced via mechanical and chemical processing of human or non-human xenogeneic tissues. The specific processing methods vary between products and may have a significant effect on the resulting mesh properties, such as mechanical strength and bioactivity. Although biologic meshes are believed to be more resistant to infection, they are often significantly more expensive than their synthetic counterparts. Parastomal hernia repairs are considered clean clean-contaminated, or contaminated repairs depending on the clinical situation. While synthetic mesh can be successfully used in clean situations, the use of biologic mesh in contaminated scenarios is beneficial. Managing infection after the use of synthetic mesh compared to biologic mesh may result in a higher rate of explantation. A recent analysis of our outcomes in patients undergoing abdominal wall reconstruction using biologic mesh demonstrates that while the presence of concomitant parastomal or stomal site hernias increases the rate of surgical site occurrence, the rate of hernia recurrence remains the same. In our cohort of patients who underwent ventral and parastomal hernia repair using biologic mesh, the rate of surgical site infection was $11 \%$, and hernia recurrence was $7 \%$ over a 27 -month median follow-up interval ${ }^{[30]}$. Most infections were managed conservatively using antibiotics and drain placement. Mesh explanation was rarely necessary ${ }^{[30]}$. More data are still needed to establish the superiority of biologic mesh over synthetic mesh in parastomal hernia repair ${ }^{[3]}$.

Degradable biosynthetic meshes have been championed for use in abdominal wall reconstruction due to the purported ability to offer tissue integration and resistance to infection at a much lower cost to healthcare systems than traditional biologic mesh ${ }^{[31]}$. These products are synthetic polymers that are enzymatically degraded and replaced by tissue ingrowth over time. Examples of commercially available biodegradable mesh include Bio- $\mathrm{A}^{\oplus}$ Tissue Reinforcement (Gore, Flagstaff, AZ, USA), TIGR ${ }^{\oplus}$ Matrix Surgical Mesh (Novus Scientific, Uppsala, Sweden), and Phasix ${ }^{\mathrm{Tw}}$ Mesh (BD Bard, Warwick RI, USA). To date, there is limited data to draw conclusions regarding the use of biodegradable mesh in abdominal wall reconstruction at large, and no studies are specifically devoted to the study of these products in parastomal hernia repair. 
Much of the available literature consists of preclinical animal histologic analysis and small population studies $^{[31-37]}$. The Complex Open Bioabsorbable Reconstruction of the Abdominal Wall (COBRA) Study evaluated fascial reinforcement of contaminated ventral hernia repairs with Bio- $\mathrm{A}^{\oplus}$ in 104 patients and demonstrated an infection rate of $18 \%$ and recurrence rate of $17 \%$ at 24 months (COBRA) ${ }^{[38]}$. Due to the aforementioned lack of data and the high baseline recurrence rate of parastomal hernias, the use of degradable mesh to reconstruct these defects is not currently recommended by the authors.

\section{Location of mesh placement}

The three main anatomic planes of mesh placement utilized during parastomal hernia repair are onlay, sublay, and underlay. The onlay technique involves securing the mesh superficially to the anterior rectus sheath and external oblique aponeurosis. This is most commonly accomplished via a cutaneous incision in close proximity to the parastomal hernia. The benefits of onlay placement include speedy and relatively simple placement, avoidance of laparotomy and ventral hernias, and reduced visceral injury rates. The drawbacks include a relatively high hernia recurrence rate and possible skin necrosis secondary to the devascularization of large cutaneous flaps when placing the mesh. The onlay technique may be associated with an increased risk of infectious complications because of the proximity of the mesh to the stomal opening, but this association has not been validated ${ }^{[39]}$. The retromuscular or sublay technique involves placing the mesh between the rectus muscle abdominus and posterior rectus sheath, which can be accomplished via an open or minimally invasive approach. The benefits of sublay placement include avoidance of visceral injury and less cutaneous risk than an onlay approach. The drawbacks include technical difficulty and violation of the hernia space ${ }^{[40]}$. The underlay technique involves intra-abdominal placement of the mesh in the preperitoneal or intraperitoneal layer, which can be accomplished via both open and minimally invasive approaches. The main benefit of the underlay technique is the lack of cutaneous risk; drawbacks include technical difficulty and risks of visceral injury secondary to the procedure and subsequent mesh-associated adhesion formation. Sublay and underlay positioning may offer a biomechanical advantage secondary to being compressed to the overlying abdominal wall musculature by underlying intra-abdominal pressure ${ }^{[22,28]}$.

\section{Open mesh-based surgical techniques}

The Sugarbaker and keyhole techniques provide the foundational basis for many modern parastomal hernia repair methods [Figure 1] ${ }^{[29]}$. The Sugarbaker technique was first published in $1985^{[41]}$. This technique involves an open approach through the initial midline or paramedian laparotomy incision. Once the incision is widely opened, Kocher clamps or self-retaining retractors are used to elevate the fascial edge. Adhesiolysis is performed, and the hernia sac is moved into the abdominal cavity. The mesh is cut to snugly fit around the fascial defect and secured with individual sutures placed at $1 \mathrm{~cm}$ intervals around the fascial ring. A small opening is left in the lateral aspect of the mesh closure through which the bowel is tunneled and secured to the overlying abdominal wall with sutures. Similar to the Sugarbaker technique, the keyhole technique utilizes an underlay mesh to reinforce the fascial defect. However, instead of lateralizing the bowel, the keyhole technique passes the bowel through a small opening in the center of the mesh. Care must be taken to ensure that the mesh opening is of the proper dimensions, as obstruction and recurrence can result from undersized and oversized openings, respectively ${ }^{[28]}$.

In 2014, Raigani et al. ${ }^{[42]}$ reported a novel open approach involving stoma site repositioning with prophylactic retromuscular mesh reinforcement using a modified keyhole technique. Of 46 patients, only 5 (11\%) developed a recurrence, with a mean follow-up time of 13 months. The benefits of this approach include the ability to identify and repair multiple defects at once and limiting exposure between the bowel and mesh; the disadvantages include morbidity associated with stoma relocation and potential alteration of 


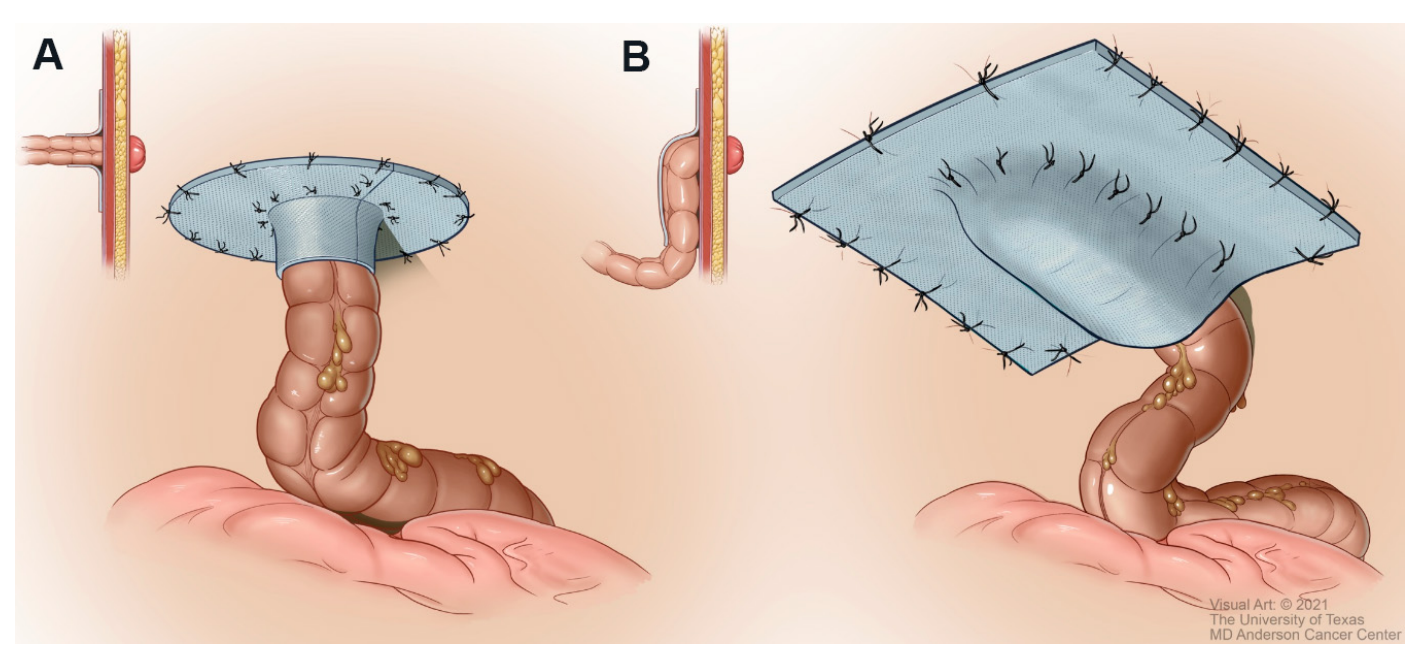

Figure 1. Mesh reinforcement of parastomal hernia defects. (A) Keyhole configuration of mesh placement. (B) Sugarbaker configuration of mesh placement.

the mechanical properties of the mesh secondary to the keyhole incision. Noting these limitations, Pauli et al.$^{[33]}$ developed a retromuscular parastomal hernia repair that combines a transversus abdominus release with a modified Sugarbaker mesh reinforcement. Although technically demanding, this technique preserves the biomechanics of the abdominal wall, provides unaltered mesh reinforcement, and avoids stoma relocation. Unfortunately, data regarding long-term patient outcomes and the widespread clinical utility of Pauli et al. ${ }^{[43]}$ 's technique are currently unavailable.

\section{Minimally invasive mesh-based surgical techniques}

The use of minimally invasive approaches for parastomal hernia repair has gradually increased over the past two decades. Although early efforts mainly focused on the laparoscopic translation of the Sugarbaker and keyhole techniques, increased experience has led to further modification of these approaches in an attempt to improve patient outcomes. In 2005, LeBlanc et al. ${ }^{[44]}$ published a variation of the keyhole technique involving two overlapping prostheses with slits oriented in opposing positions to prevent herniation of the bowel through the slit in the first mesh. The two-mesh approach was further refined in 2010 when Berger ${ }^{[45]}$ reported using the sandwich technique, a laparoscopic combination of the keyhole and Sugarbaker techniques. This approach involves passing the bowel through a piece of intraperitoneal mesh (i.e., the keyhole technique) and then using a second piece of mesh to lateralize the bowel and secure it to the overlying abdominal wall (i.e., the Sugarbaker technique).

While the majority of published studies on minimally invasive parastomal hernia repair have focused on variations of the Sugarbaker, keyhole, or sandwich techniques, there is growing evidence supporting the use of robotic surgery to repair these defects. Maciel et al. ${ }^{[46]}$ successfully utilized a robotic approach to perform retromuscular keyhole parastomal hernia repair in two patients. In 2020, Formisano et al. ${ }^{[47]}$ published a novel robotic technique combining retromuscular mesh reinforcement and posterior component separation with transversus abdominus release. While the long-term success and overall cost-effectiveness of robotic parastomal hernia repair remain unclear, these approaches show promise and will likely become more prevalent. 


\section{OUTCOMES}

Parastomal hernia repair is a reconstructive challenge with historically poor outcomes. While the desire to solve this clinical problem has been the impetus for substantial surgical innovation, the resulting amount of procedural variability complicates comparative analyses. This complication is further convoluted by a historical lack of standardization regarding hernia classification and differences in the duration of follow-up between studies.

In a systematic review of 30 studies, Hansson et al. ${ }^{[39]}$ concluded that parastomal hernias repaired with sutures alone were associated with a 9-fold higher recurrence rate compared to those repaired using mesh reinforcement $(\mathrm{OR}=8.9 ; 95 \% \mathrm{CI}$ : 5.2-15.1; $P<0.0001)$. There were no significant differences between mesh techniques with regard to infection rates, post-operative morbidity, and recurrence rates. Open and laparoscopic procedures did not significantly differ with respect to morbidity, recurrence, or duration of the operation. Amongst laparoscopic procedures, hernias repaired with the keyhole technique are associated with a two-fold increase in recurrence compared to those repaired with the Sugarbaker technique $(\mathrm{OR}=2.3$; 95\%CI: 1.2-4.6; $P=0.016)$. In Hansson et al. ${ }^{[39]}$ 's review, only studies with at least 12 months of follow-up were included in the pooled analysis for calculating recurrence rates (reported mean and median range, 1278 months).

Hotouras et al. ${ }^{[48]}$ analyzed 115 studies and found no significant differences in reported complications between onlay (recurrence, 0.0\%-62.5\%; infection, 0.0\%-28.5\%; erosion, 0.0\%-6.2\%; removal, 0.0\%-22.7\%), intraperitoneal (recurrence, $0.0 \%-28.6 \%$; infection, $0.0 \%-14.3 \%$; erosion, $0.0 \%$; removal, $0.0 \%-14.3 \%$ ), and subperitoneal (recurrence, $0.0 \%-28.6 \%$; infection, $0.0 \%-20.0 \%$; erosion, $0.0 \%$; removal, $0.0 \%-10 \%$ ) mesh placement. Laparoscopic repairs were found to have similar outcomes to open procedures (recurrence, 0.0\%-46.4\%; infection, 0.0\%-16.6\%; erosion, 0.0\%-1.5\%; removal, $0.0 \%-9.5 \%$ ). The reported mean and median follow-up durations of the included studies ranged from 6 to over 96 months, with the majority of studies having greater than two years of follow-up.

Mericli et al. ${ }^{[30]}$ evaluated the outcomes of patients undergoing combined ventral and parastomal hernia repair (abdominal wall reconstruction + ostomy, 118 patients) compared to ventral hernia repair alone (abdominal wall reconstruction, 381 patients). The authors found a significantly higher percentage of surgical site occurrences (defined as one or more instances of hematoma, seroma, skin dehiscence, or fat necrosis) in the abdominal wall reconstruction + ostomy cohort compared to the abdominal wall reconstruction cohort ( $34.1 \%$ vs. $17.7 \%$; adjusted $\mathrm{OR}=2.3$; 95\%CI: 1.4-3.7; $P<0.001$ ). However, there was no significant difference in the rate of ventral hernia recurrence between the two cohorts before $(8.7 \% v s$. $6.8 \%, P=0.60)$ and after $(7.7 \%$ vs. $6.6 \%, P>0.99)$ propensity score matching for confounding variables ${ }^{[30]}$. The median reported follow-up duration was 27.2 months (interquartile range, 12.4-46 months).

DeAsis et al ${ }^{[49]}$ performed a meta-analysis of 15 articles comparing laparoscopic parastomal hernia repair. The authors reported an overall surgical site infection rate of 3.8\%, mesh infection rate of $1.7 \%$, obstruction rate of $1.7 \%$, and overall recurrence rate of $17.4 \%$. When the recurrence rate was stratified according to technique, the Sugarbaker technique was associated with a lower recurrence rate than was the keyhole approach (10.2\% vs. 27.9\%). All included studies had at least 12 months of follow-up (reported mean and median range, 13-36 months). The authors reported that $3.1 \%$ of patients underwent conversion from a laparoscopic to an open approach.

Large prospective clinical trials to facilitate a greater understanding of the technical factors associated with successful outcomes would be of immense value, although the logistic feasibility of this task is daunting in 
light of the number of procedural variables in a medically complex patient population.

\section{AUTHORS' APPROACH}

- Use prior laparotomy incision to expose the hernia, perform lysis of adhesions, and reduce hernia contents.

- Avoid re-siting (moving stoma to a new location on the abdominal wall) unless the adjacent fascia is significantly compromised, and re-siting is absolutely necessary.

- Reduce the size of the fascial trephine in transverse orientation using interrupted \#1 polydioxanone or \#1 polypropylene sutures.

- Use non-human xenogeneic biologic mesh for underlay reinforcement.

- Use modified Sugarbaker reinforcement if the mesentery length is adequate.

- Use a keyhole or cruciate mesh design if the mesentery length is inadequate.

- If the keyhole or cruciate technique is used, consider suturing the mesh edges loosely to the bowel serosa with shallow bites.

- Extend the mesh to reinforce the remaining ventral abdominal wall if needed.

- Postoperative care/restrictions.

$\circ$ Avoid lifting weights greater than 15lbs for three weeks.

- Wear abdominal binder for at least three to six months. In certain cases the binder can be modified to include a space for the ostomy bag.

$\circ$ After six months, wear binder when engaging in strenuous activities.

$\circ$ Avoid submersion of the incision underwater for 6 weeks (tub-bathing, swimming, etc.).

\section{CONCLUSIONS}

Parastomal hernias are complex conditions that can be repaired in a variety of ways. Currently, there is an overall lack of data available to identify a superlative surgical approach. Given this, an understanding of the relative risks and benefits of all available techniques is needed to formulate a management plan that maximizes safety and the likelihood of long-term reconstructive success. Further research is needed to facilitate a long-term comparative analysis of the techniques described in this review.

\section{DECLARATIONS}

\section{Acknowledgements}

We thank Ann Sutton, Scientific Editor, and Ashli Nguyen-Villarreal, Associate Scientific Editor, in the Research Medical Library at The University of Texas MD Anderson Cancer Center, for editing this article. 


\section{Authors' contributions}

Participated in the accumulation of data, literature review, and writing and editing of this manuscript: Smith JM, Kapur SK, Mericli AF, Baumann DP, Butler CE

\section{Availability of data and materials}

Not applicable.

\section{Financial support and sponsorship}

None.

\section{Conflicts of interest}

All authors declared that there are no conflicts of interest.

\section{Ethical approval and consent to participate}

Not applicable.

\section{Consent for publication}

Not applicable.

\section{Copyright}

(C) The Author(s) 2022.

\section{REFERENCES}

1. Styliński R, Alzubedi A, Rudzki S. Parastomal hernia - current knowledge and treatment. Wideochir Inne Tech Maloinwazyjne 2018;13:1-8. DOI PubMed PMC

2. Goligher J. Surgery of the anus, colon and rectum. 5th ed. London: Bailliere Tindall; 1984. p. 703-4

3. Antoniou SA, Agresta F, Garcia Alamino JM, et al. European Hernia Society guidelines on prevention and treatment of parastomal hernias. Hernia 2018;22:183-98. DOI PubMed

4. Pilgrim $\mathrm{CH}$, McIntyre R, Bailey M. Prospective audit of parastomal hernia: prevalence and associated comorbidities. Dis Colon Rectum 2010;53:71-6. DOI PubMed

5. Sohn YJ, Moon SM, Shin US, Jee SH. Incidence and risk factors of parastomal hernia. J Korean Soc Coloproctol 2012;28:241-6. DOI PubMed PMC

6. Zhao F, Chen F, Yuan X, Liu Y, Chen J. Decreased collagen type III synthesis in skin fibroblasts is associated with parastomal hernia following colostomy. Int J Mol Med 2019;44:1609-18. DOI PubMed PMC

7. Tsujinaka S, Tan KY, Miyakura Y, et al. Current management of intestinal stomas and their complications. J Anus Rectum Colon 2020;4:25-33. DOI PubMed PMC

8. Shabbir J, Chaudhary BN, Dawson R. A systematic review on the use of prophylactic mesh during primary stoma formation to prevent parastomal hernia formation. Colorectal Dis 2012;14:931-6. DOI PubMed

9. Sjödahl R, Anderberg B, Bolin T. Parastomal hernia in relation to site of the abdominal stoma. Br J Surg 1988;75:339-41. DOI PubMed

10. Strong SA. The difficult stoma: challenges and strategies. Clin Colon Rectal Surg 2016;29:152-9. DOI PubMed PMC

11. Nguyen AT, Thompson KM, Baumann DP, Butler CE. Urinary conduits do not preclude the use of the ipsilateral vertical rectus abdominis myocutaneous (VRAM) flap. Plast Reconstr Surg 2015;135:1200. DOI

12. Lian L, Wu XR, He XS, et al. Extraperitoneal vs. intraperitoneal route for permanent colostomy: a meta-analysis of 1,071 patients. Int $J$ Colorectal Dis 2012;27:59-64. DOI PubMed

13. Kroese LF, de Smet GH, Jeekel J, Kleinrensink GJ, Lange JF. Systematic review and meta-analysis of extraperitoneal versus transperitoneal colostomy for preventing parastomal hernia. Dis Colon Rectum 2016;59:688-95. DOI PubMed

14. Cross AJ, Buchwald PL, Frizelle FA, Eglinton TW. Meta-analysis of prophylactic mesh to prevent parastomal hernia. Br J Surg 2017;104:179-86. DOI PubMed

15. Odensten C, Strigård K, Rutegård J, et al. Use of prophylactic mesh when creating a colostomy does not prevent parastomal hernia: a randomized controlled trial - STOMAMESH. Ann Surg 2019;269:427-31. DOI

16. Liedberg F, Kollberg P, Allerbo M, et al. Preventing parastomal hernia after ileal conduit by the use of a prophylactic mesh: a randomised study. Eur Urol 2020;78:757-63. DOI PubMed

17. Cingi A, Cakir T, Sever A, Aktan AO. Enterostomy site hernias: a clinical and computerized tomographic evaluation. Dis Colon Rectum 2006;49:1559-63. DOI PubMed

18. de Smet GHJ, Lambrichts DPV, van den Hoek S, et al. Comparison of different modalities for the diagnosis of parastomal hernia: a 
systematic review. Int $J$ Colorectal Dis 2020;35:199-212. DOI PubMed

19. Gurmu A, Matthiessen P, Nilsson S, Påhlman L, Rutegård J, Gunnarsson U. The inter-observer reliability is very low at clinical examination of parastomal hernia. Int J Colorectal Dis 2011;26:89-95. DOI PubMed

20. Śmietański M, Szczepkowski M, Alexandre JA, et al. European Hernia Society classification of parastomal hernias. Hernia 2014;18:16. DOI PubMed PMC

21. Okorji LM, Kasten KR. Diagnosis and management of parastomal hernias. Dis Colon Rectum 2019;62:158-62. DOI PubMed

22. Shakarchi J, Williams JG. Systematic review of open techniques for parastomal hernia repair. Tech Coloproctol 2014;18:427-32. DOI PubMed

23. Roussel B, Mulieri G, Gauzolino R, Danion J, De Wailly P, Faure JP. Parastomal hernia. J Visc Surg 2012;149:e15-9. DOI PubMed

24. Carne PW, Robertson GM, Frizelle FA. Parastomal hernia. Br J Surg 2003;90:784-93. DOI PubMed

25. Köhler G, Mayer F, Wundsam H, Schrittwieser R, Emmanuel K, Lechner M. Changes in the surgical management of parastomal hernias over 15 years: results of 135 cases. World J Surg 2015;39:2795-804. DOI PubMed

26. Reinforcement of Closure of Stoma Site (ROCSS) Collaborative and West Midlands Research Collaborative. Prophylactic biological mesh reinforcement versus standard closure of stoma site (ROCSS): a multicentre, randomised controlled trial. Lancet 2020;395:41726. DOI

27. Rosin JD, Bonardi RA. Paracolostomy hernia repair with Marlex mesh: a new technique. Dis Colon Rectum 1977;20:299-302. DOI PubMed

28. Glasgow SC, Dharmarajan S. Parastomal hernia: avoidance and treatment in the 21st century. Clin Colon Rectal Surg 2016;29:277-84. DOI PubMed PMC

29. Gillern S, Bleier JI. Parastomal hernia repair and reinforcement: the role of biologic and synthetic materials. Clin Colon Rectal Surg 2014;27:162-71. DOI PubMed PMC

30. Mericli AF, Garvey PB, Giordano S, Liu J, Baumann DP, Butler CE. Abdominal wall reconstruction with concomitant ostomyassociated hernia repair: outcomes and propensity score analysis. J Am Coll Surg 2017;224:351-361.e2. DOI PubMed

31. Keogh K, Slater K. Comparison of biosynthetic versus synthetic mesh in clean and contaminated ventral hernia repairs. ANZ J Surg 2020;90:542-6. DOI PubMed

32. Finch D, Mehmood S, Varghese J. Abdominal wall reconstruction using biosynthetic absorbable mesh in high-risk complex ventral hernia. Swiss Med Wkly 2021;151:w20449. DOI PubMed

33. Peeters E, van Barneveld KW, Schreinemacher MH, et al. One-year outcome of biological and synthetic bioabsorbable meshes for augmentation of large abdominal wall defects in a rabbit model. J Surg Res 2013;180:274-83. DOI PubMed

34. Deeken CR, Matthews BD. Characterization of the mechanical strength, resorption properties, and histologic characteristics of a fully absorbable material (Poly-4-hydroxybutyrate-PHASIX Mesh) in a porcine model of hernia repair. ISRN Surg 2013;2013:238067. DOI PubMed PMC

35. Parker MJ, Kim RC, Barrio M, et al. A novel biosynthetic scaffold mesh reinforcement affords the lowest hernia recurrence in the highest-risk patients. Surg Endosc 2021;35:5173-8. DOI PubMed

36. Hjort H, Mathisen T, Alves A, Clermont G, Boutrand JP. Three-year results from a preclinical implantation study of a long-term resorbable surgical mesh with time-dependent mechanical characteristics. Hernia 2012;16:191-7. DOI PubMed PMC

37. Rognoni C, Cuccurullo D, Borsoi L, et al. Clinical outcomes and quality of life associated with the use of a biosynthetic mesh for complex ventral hernia repair: analysis of the "Italian Hernia Club" registry. Sci Rep 2020;10:10706. DOI PubMed PMC

38. Rosen MJ, Bauer JJ, Harmaty M, et al. Multicenter, prospective, longitudinal study of the recurrence, surgical site infection, and quality of life after contaminated ventral hernia repair using biosynthetic absorbable mesh: the COBRA study. Ann Surg 2017;265:205-11. DOI PubMed PMC

39. Hansson BM, Slater NJ, van der Velden AS, et al. Surgical techniques for parastomal hernia repair: a systematic review of the literature. Ann Surg 2012;255:685-95. DOI PubMed

40. Techagumpuch A, Udomsawaengsup S. Update in parastomal hernia. Ann Laparosc Endosc Surg 2019;4:75. DOI

41. Sugarbaker PH. Peritoneal approach to prosthetic mesh repair of paraostomy hernias. Ann Surg 1985;201:344-6. DOI PubMed PMC

42. Raigani S, Criss CN, Petro CC, Prabhu AS, Novitsky YW, Rosen MJ. Single-center experience with parastomal hernia repair using retromuscular mesh placement. J Gastrointest Surg 2014;18:1673-7. DOI PubMed

43. Pauli EM, Juza RM, Winder JS. How I do it: novel parastomal herniorrhaphy utilizing transversus abdominis release. Hernia 2016;20:547-52. DOI PubMed

44. LeBlanc KA, Bellanger DE, Whitaker JM, Hausmann MG. Laparoscopic parastomal hernia repair. Hernia 2005;9:140-4. DOI PubMed

45. Berger D. [Laparoscopic repair of parastomal hernia]. Chirurg 2010;81:988-92. DOI PubMed

46. Maciel V, Mata W, Arevalo G, Zeichen M, Glass T. Robotic retro-rectus repair of parastomal hernias. J Robot Surg 2019;13:483-9. DOI PubMed

47. Formisano G, Giuliani G, Salvischiani L, Salaj A, Bianchi PP. Robotic repair of a parastomal hernia with transversus abdominis release - a video vignette. Colorectal Dis 2020;22:222-3. DOI PubMed

48. Hotouras A, Murphy J, Thaha M, Chan CL. The persistent challenge of parastomal herniation: a review of the literature and future developments. Colorectal Dis 2013;15:e202-14. DOI PubMed

49. DeAsis FJ, Lapin B, Gitelis ME, Ujiki MB. Current state of laparoscopic parastomal hernia repair: a meta-analysis. World $J$ Gastroenterol 2015;21:8670-7. DOI PubMed PMC 